\title{
Effect of Administration of Acrylamide and Possible Protective Role of Vitamin E on Postnatal Rat Liver Structure
}

\section{Hegazy AA*, Morsy MM, Shehata MA and Agaga RA}

Anatomy and Embryology Department, Faculty of Medicine, Zagazig University, Egypt

*Corresponding author: Abdelmonem A Hegazy, Anatomy and Embryology Department, Faculty of Medicine, Zagazig University, Egypt, Email: dr.abdelmonemhegazy@yahoo.com

\section{Research Article \\ Volume 2 Issue 2}

Received Date: August 17, 2018

Published Date: October 05, 2018

\section{Abstract}

Aim of Study: This study aimed to reveal the structural changes occurring in the liver of albino rats in the postnatal ages after oral administration of acrylamide (ACR) to their pregnant mothers and also to evaluate the protective role of vitamin $\mathrm{E}(\mathrm{VE})$.

Methods: Fifty-four pregnant rats were equally divided into three groups: control; ACR-treated (received 10mg/kg/day by gastric gavage) and ACR+VE treated group (received ACR plus vitamin E, 100mg/kg/day daily). Treatment was given from day 7 of gestation till weaning. Pups were divided into two subgroups; postnatal days 1 and 21 (D1 and D21). Liver was processed for hematoxylin and eosin (H\&E), Mallory stain, caspase 3 and electron microscopic (EM) examination. The mean area percent of collagen fibers and positive caspase 3 reactions were measured.

Results: The ACR-treated subgroups at D1 and D21 revealed degenerative changes of hepatocytes. There were congested dilated central and portal veins, bile duct proliferation, marked increase of collagen fibers, strong positive reaction for Caspase 3 and electron-lucent areas of the cytoplasm of hepatocytes, with many vacuoles and shrunken electron-dense nuclei. On the other hand, the protective group receiving ACR plus VE showed some improvement of the histological and morphometric findings.

Conclusion: Acrylamide caused marked hazardous impact in the liver of postnatal albino rats. Fortunately, concomitant administration of vitamin E might protect the liver.

Keywords: Liver; Rat; Acrylamide; Vitamin E; Toxicity Protection

Abbreviations: ACR: Acrylamide; VE: Vitamin E; D1: First Postnatal Day; D21: Postnatal Day 21; D1ACR: Animals Treated with Acrylamide and Investigated at the First Postnatal Day; D21ACR: Animals Treated with Acrylamide at the Postnatal Day 21; D1ACRVE: Animals Treated with Acrylamide and Concomitant VE at the First Postnatal Day; D21ACRVE: Animals Treated with Acrylamide and Concomitant VE at the Postnatal Day 21;
H\&E: Hematoxylin and Eosin; LM: Light Microscopy; EM: Electron Microscopy; ANOVA: Analysis of Variance; SD: Standard Deviation; LSD: Least Significant Difference.

\section{Introduction}

Liver is considered as the predominant site for detoxifying and biotransformation of toxic substances 


\section{Journal of Embryology \& Stem Cell Research}

reaching the body; it regulates the blood concentration of many metabolites, particularly glucose and amino acids. It has also a great role in synthesizing, storing, secreting, regulating, transforming and breaking down of many materials in the body [1].

It is responsible for detoxifying of several drugs in addition to a great variety of industrial solvents. Attributing to its functional reserve, the liver can suffer extensive destruction or damage before malfunction becomes observed [2].

Acrylamide (ACR) is a widely distributed substance that has several chemical and industrial applications such as cosmetic substances, tobacco industry and waste water management or processing. In addition, ACR is used extensively for gel chromatography in molecular laboratories [3]. ACR is formed in starchy foods that are roasted, baked or fried at high temperature such as chips, fried potatoes, toasted bread toasted coffee particles and baked product like biscuits [4]. Although ACR accidental exposure can occur from many occupations [5] still the main principal route of human exposure is via the diet [6].

The reactive oxygen species (ROS) are produced by natural physiological processes. ROS perform important functions at low or moderate concentrations. Imbalance between the destructive ROS production and natural cellular antioxidant defenses is the primary cause of oxidative stress. Antioxidants are the key stone in the defense mechanism against oxidative stress produced by free radicals [7].

The oxidative stress induced by ACR occurs via affection of lipid peroxidation in addition to reduction of catalase enzyme and glutathione (GSH) [8]. Histopathological investigations declared that chronic ACR administration produces degeneration, apoptosis and necrosis in the liver tissue of ACR-treated rats [9]. On the other hand, vitamin $\mathrm{E}$ (VE) is a lipid-soluble compound, abundant in certain food like vegetables and oil. It is a potent chain breaking antioxidant that acts to destruct reactive oxygen and nitrogen species [10].

The aim of this work was to elucidate the structural changes occurring in the liver of albino rats at the postnatal ages after toxic oral administration of ACR to their pregnant mothers and also to evaluate the possible protective role of VE against ACR toxicity by using light (LM) and electron microscopic (EM) examinations as well as immunohistochemical and morphometric investigations.

\section{Materials and Methods}

\section{Chemicals}

Acrylamide: ACR of purity > 99\% was dissolved in distilled water and orally administered by gastric gavage at a dose of $10 \mathrm{mg} / \mathrm{kg} /$ day [11].

Vitamin E: VE in the form of powder ( $\alpha$-tocopherol) was dissolved in distilled water and given orally by gastric gavage at a dose of $100 \mathrm{mg} / \mathrm{kg} /$ day [12]. The ACR and VE were obtained from El Gomhoria Company for Chemical and Medical Trading, Zagazig, Egypt.

\section{Animals}

A total of 81 albino rats were used in this study. Fiftyfour adult females and 27 adult males weighing 140-150 g were purchased from the animal house. Females were housed with males at ratio of 2:1 respectively in each cage. The day of detecting sperms in the vaginal smear was considered as day one of gestation. Pregnant rats were equally divided into three groups, each comprised 18 pregnant animals; and their off spring were examined at postnatal days 1 (D1) and 21 (D21). Group 1 (control) contained 18 pregnant rats; 9 rats were given distilled water orally (control negative) and the others received distilled water by gastricavage and considered as a (control positive) group. Group 2 (ACR-treated) was given ACR daily from the day 7 of gestation till weaning. Third (protected) group was given ACR plus VE daily from day 7 of gestation till weaning. Weaning age of the rats was three weeks [13].

At birth, each mother was housed with its pups in a large cage. The pubs were examined at two postnatal days; the first and day 21 . So each group was divided into two subgroups as follows:

This group contained 40 pups with their mothers not exposed to chemicals. The animals were divided into four subgroups each contained 10 rats:

i. Subgroup D1 (control negative) andD1*(control positive).

ii. Subgroup D21 (control negative) andD21*(control positive).

Group 2: This group contained 20pups; their mothers were exposed to ACR. This group was divided into two subgroups, each contained 10rats; D1ACR andD21ACR at the postnatal days; one and 21 respectively. 


\section{Journal of Embryology \& Stem Cell Research}

Group 3: This group contained 20 pups of their mothers exposed to ACR plus VE. This group was divided into two subgroups, each contained 10rats: D1ACRVE and D21ACRVE at the postnatal days; one and 21 days respectively.

Rats from each group were anaesthetized, scarified at the previously defined days then dissected for removal of their liver.

\section{Histological Study}

The liver from each animal was carefully dissected were immediately immersed in $10 \%$ formol saline for 12 hours, then processed for light microscopic examination [14] and subjected to hematoxylin and eosin (H\&E) staining and Mallory' strichrome stain [15].

\section{Immunohistochemical Stain 'Anticaspase 3'}

According to Bancroft and Gamble [15], a rabbit monoclonal antibody of IgG type was carried out for localization of caspase 3 (apoptosis marker) in paraffin sections.

\section{EM Study}

Liver tissue was cut into small pieces $1 \mathrm{~mm}^{3}$ each using sharp blade razor. These pieces were immersed in phosphate-buffered gluteraldehyde (2.5\%) for one day and processed for EM investigation [16]. Ultrathin sections were examined and photographed by transmission electron microscope (JEOL TEM 1010) in Electron Microscope Research Laboratory.

\section{Morphometric Study}

Sections stained for caspase 3 and Mallory's trichrome were morphometrically analyzed. The area percentage of collagen fibers and area percentage of immune reaction to caspase 3 were measured using the Fiji Image $\mathrm{J}(1.51 \mathrm{n}, \mathrm{NIH}, \mathrm{USA})$ program.

\section{Statistical Analysis}

Statistical analysis was performed for the area percentage of collagen fibers and area percentage of immune reaction to caspase3. All the values of experiments were represented as mean \pm standard deviation (SD). One-way analysis of variance (ANOVA) was used, followed by least significant difference (LSD) test to evaluate the differences between the groups. For all comparison $\mathrm{P}<0.05$ were considered as significant difference. All analyses were performed using the IBM SPSS 18.0 software [17].

\section{Results}

\section{Control Groups}

H\&E Staining: In all different age groups the positive and negative control groups were similar to each other, so the negative control results were representative of all control groups. Light microscopic examination of sections from the liver of first postnatal day (D1) control subgroup showed scattered erythroid islands (small islands of hematopoietic cells). The erythroid cells were characterized by intense hyperchromasia in their nuclei in contrast to pale nuclei of hepatocytes. The hepatocytes were normal with characteristic polygonal large vesicular nuclei and acidophilic cytoplasm. Some binucleated hepatocytes were seen. LM examination of sections from the liver of D21control subgroup showed absence of erythroid islands and nearly characteristic hepatic lobule where polyhedral hepatocytes radiating from central vein. Some binucleated hepatocytes were observed. The hepatocytes were separated by normal blood sinusoids with Kupffer cells in the irlining. Portal areas are present at the corners containing portal vein, hepatic artery and bile duct (Figure 1).
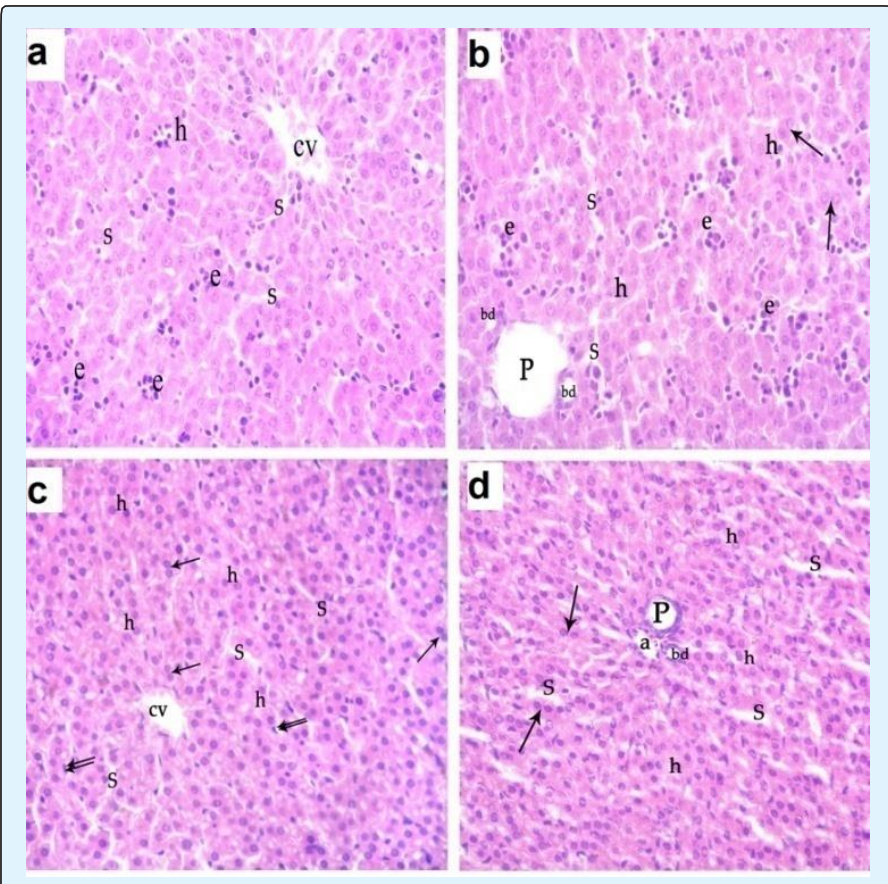

Figure 1: A photomicrograph of a liver section of control groups: (a) D1 showing normal sized central vein (cv), multiple small sized blood sinusoids(s), normal hepatocytes (h) with polygonal large vesicular nuclei and acidophilic cytoplasm. Multiple erythroid islands (e) with intense hyperchromatic nuclei are seen. (b) D1 showing 


\section{Embryology \& Stem Cell Research}

portal vein (P), normal bile duct (bd), multiple blood sinusoids (s), normal hepatocytes (h), multiple erythroid islands (e) and some binucleated hepatocytes (arrow) are seen. (c) D21 showing normal sized central vein (cv), multiple blood sinusoids (s), normal hepatocytes (h). Some binucleated hepatocytes (arrow) are seen. Notice Kupffer cell (double arrows) in the lining of blood sinusoids. (d) D21 showing normal sized portal vein (p), bile duct (bd), hepatic artery (a) multiple blood sinusoids (s), normal hepatocytes (h). Some binucleated hepatocytes (arrow) are seen. (H\&E X400).

Mallory Staining: Mallory stained sections of liver of D1andD21control subgroup s revealed few blue colored stained collagen fibers around the central vein, the lining of blood sinusoids and in the portal area (Figure 2).
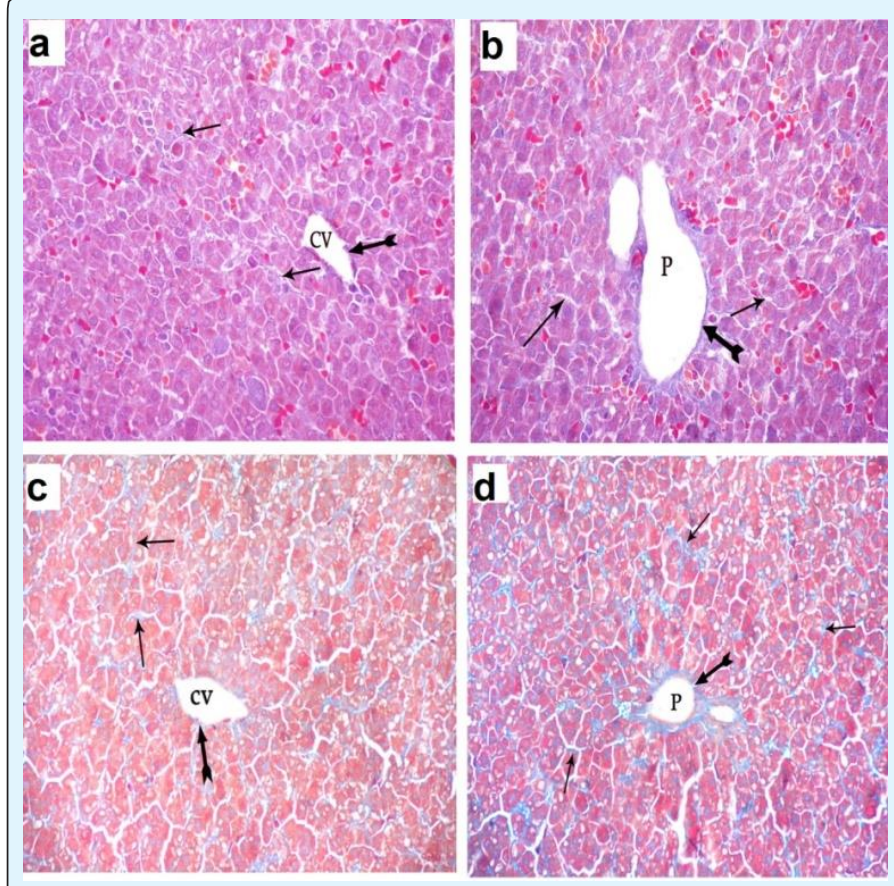

Figure 2: A photomicrograph of a liver section of control groups: (a,b) D1, (c,d) D21 showing few blue colored collagen fibers (bifid tailed arrow) around the central vein (CV), around portal vein (P) (bifid tailed arrow) and also in the lining of blood sinusoid (arrow) (Mallory stain $\mathrm{X} 400)$.

Immunohistochemical Staining for Caspase 3: Liver sections of all control subgroups'D1 and D21' showed negative immune reaction for caspase 3in the cytoplasm of hepatocytes (Figure 3).

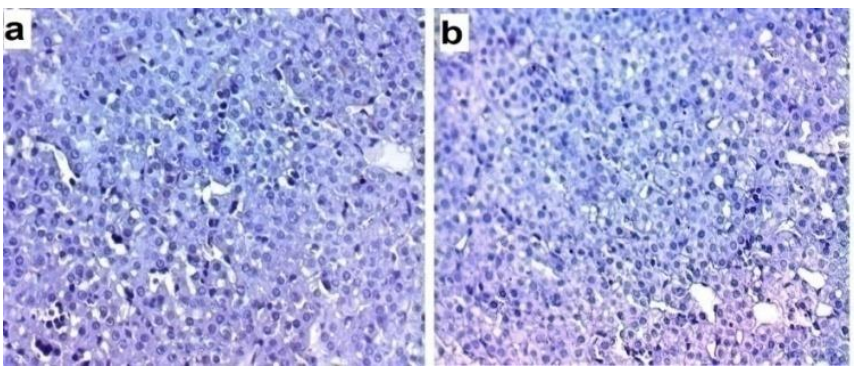

Figure 3: A photomicrograph of a liver section of control groups: (a) D1 and (b) D21 showing negative cytoplasmic immune reaction for caspase 3 (arrow). (Immuno peroxidase technique for caspase 3 X 400).

EM Examination: EM study of D1control subgroup showed that the hepatocytes appeared with euchromatic nuclei and intact nuclear envelope. The cytoplasm contained numerous mitochondria, rough endoplasmic reticulum, some lipid droplets and glycogen granules. The hepatocytes were adjacent to each other with bile canaliculi containing microvilli and occluding junctions were observed. Also normal appearance of hepatocytes was detected in D21 (Figure 4).

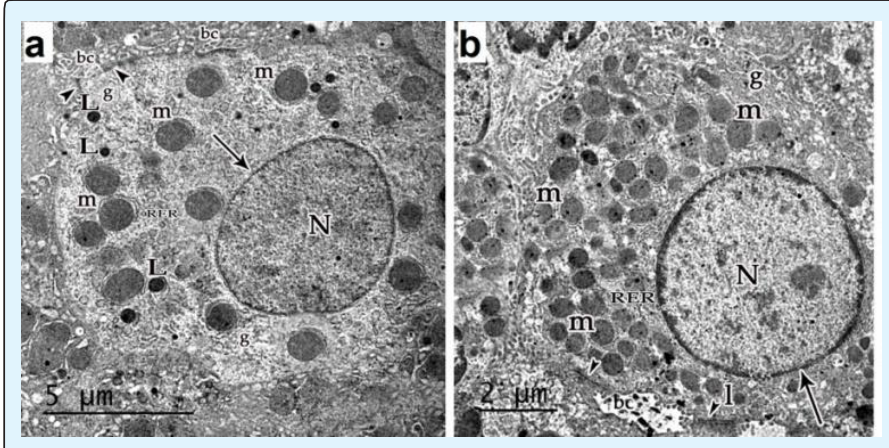

Figure 4: An EM micrograph of a section in the liver of control groups: (a) D1 showing normal hepatocyte with euchromatic nucleus $(\mathrm{N})$ and intact nuclear envelope (arrow). The cytoplasm contains many mitochondria (m), rough endoplasmic reticulum (RER), lipid droplets (L) and glycogen granules (g). Bile canaliculus (bc) with intact microvilli and occluding junctions (head arrow) are seen.(X 10200); (b) D21 showing normal hepatocyte with euchromatic nucleus (n) and intact nuclear envelope (arrow).The cytoplasm contains many mitochondria (m), rough endoplasmic reticulum (RER), lipid droplets (I) and glycogen granules (g). Bile canaliculus (bc) with intact microvilli and occluding junctions (head arrow) are seen (X 13600).

\section{2-Acrylamide-Treated Group}

Hegazy AA, et al. Effect of Administration of Acrylamide and Possible Protective Role of Vitamin E on Postnatal Rat Liver Structure. J Embryol Stem Cell Res 2018, 2(2): 000114. 


\section{Journal of Embrvologv \& Stem Cell Research}

H\&E Staining: Examination of liver sections of ACRtreated subgroup (D1ACR) showed change of normal hepatic structure. Hepatocytes had pale vacuolated cytoplasm with deeply stained shrunken nuclei. Dilated and congested central vein and blood sinusoids were observed. Scattered erythroid islands with intense hyperchromasia were present. Dilated congested Portal vein with proliferation of bile can aliculi and multiple inflammatory cellular infiltrations in portal area were seen. Liver sections of ACR-treated subgroup (D21ACR) showed more destruction of hepatic tissue. The hepatocytes were ballooned and had pale vacuolated cytoplasm with deeply stained shrunken nuclei. Dilated and congested central vein, blood sinusoids and Portal vein were more prominent. Discontinuity of endothelial lining of portal veins was noticed. Proliferated bile duct and thickened hepatic artery were seen (Figure 5).

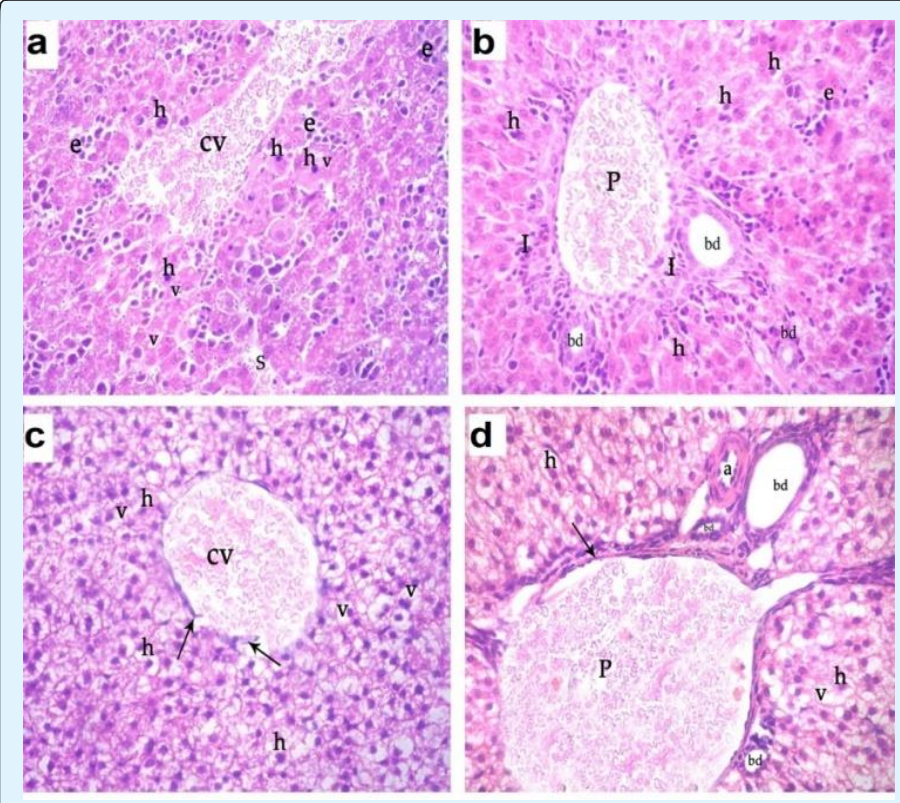

Figure 5: A photomicrograph of a liver section of ACRtreated groups: (a) D1ACR showing dilated congested central vein (cv), dilated congested blood sinusoids(s), abnormal hepatocytes (h) with vacuolated cytoplasm (v). Multiple erythroid islands (e) with intense hyperchromatic nuclei are noticed. (b) D1ACR showing dilated portal vein (p), abnormal hepatocytes (h) with vacuolated cytoplasm $(\mathrm{v}$, multiple inflammatory cellular infiltration in the portal area (I), proliferated bile duct (bd) and multiple erythroid islands (e). (c) D21ACR showing dilated central vein (cv) with discontinuity of its endothelial lining (arrow), abnormally ballooned hepatocytes (h) with dark nuclei and vacuolated cytoplasm (v). (d) D21ACR showing dilated congested portal vein (p) with thickened endothelial lining (arrow), thickened hepatic artery (a), proliferated bile duct (bd), abnormal hepatocytes $(\mathrm{h})$ with small dark stained nuclei and vacuolated cytoplasm (v). (H\&E X400).

Mallory Staining: Mallory-stained sections of D1ACR subgroup showed excess deposited collagen fibers around the central veins, blood sinusoids and portal area.InD21ACR subgroup, the liver showed more increased deposition of collagen fibers around the central veins, blood sinusoids and portal area (Figure 6).



Figure 6: A photomicrograph of a liver section of ACRtreated groups: (a) D1ACR showing mild increased blue colored collagen fibers (bifid tailed arrow) around the central vein (CV) and also in the lining of blood sinusoid (arrow). (b) D1ACR showing increased amount of blue colored collagen fibers (arrow) in the portal area. (c,d) D21ACR showing increased amount of blue colored collagen fibers (bifid tailed arrow) around the central vein $(\mathrm{CV})$, in in the portal area (P) (bifid tailed arrow) and also in the lining of blood sinusoid (arrow). (Mallory stain $\mathrm{X} 400$ ).

Immunohistochemical Staining: Liver from D1ACRsubgroup showed many positive reactions for activated caspase 3 in cytoplasm of hepatocytes. Liver of D21ACR subgroup showed extensive positive reaction for activated caspase3 more than D1 in the cytoplasm of hepatocytes (Figure 7). 


\section{Journal of Embryology \& Stem Cell Research}

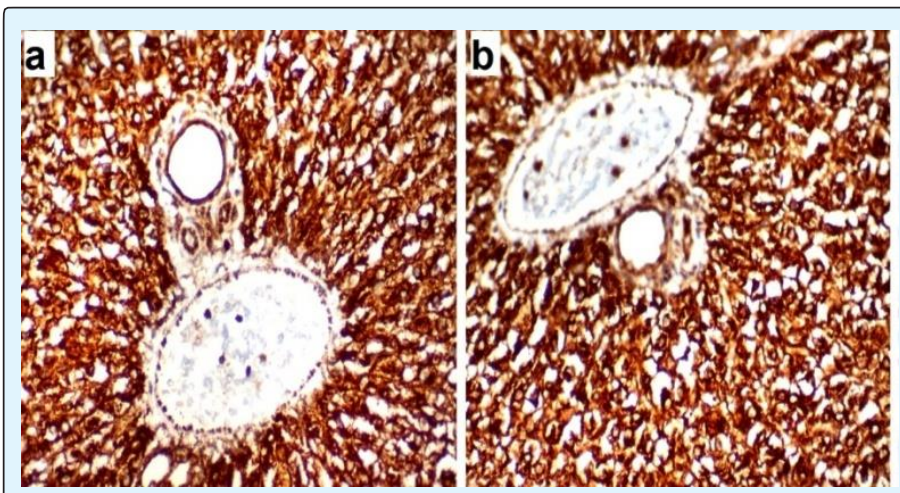

Figure 7: A photomicrograph of a liver section of ACRtreated groups: (a) D1ACR and (b) D21ACR showing marked expression of cytoplasmic immune reaction for caspase 3 of hepatocytes. (Immunoperoxidase technique for caspase $3 \mathrm{X} 400$ ).

EM Examination: Liver section of D1ACR subgroup showed abnormal hepatocyte with indented nucleus, peripheral condensation of chromatin and discontinuous nuclear envelope. The cytoplasm contained abnormally swollen mitochondria, few rough endoplasmic reticulum, lipid droplets, many vacuoles and glycogen granules. Bile can aliculus was dilated. Kupffer cell contained numerous vesicles and lysosomes varying in size and shape in the cytoplasm were found. In D21ACR subgroup, the hepatocytes appeared abnormal with shrunken nucleus and peripheral condensation of chromatin. The cytoplasm contained abnormal shaped elongated mitochondria, rough endoplasmic reticulum and some lipid droplets. Rarefication of the cytoplasm due to destruction of cytoplasmic organelles appeared as electron lucent areas. Bile can aliculus contained few microvilli Figure 8.

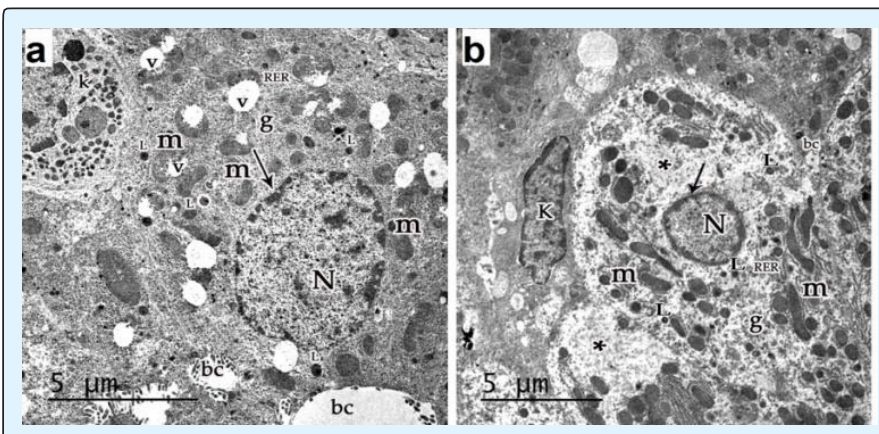

Figure 8: An EM micrograph of a section in the liver of ACR-treated groups: (a) D1ACR showing abnormal hepatocyte with indented nucleus $(\mathrm{N})$ with peripheral condensation of chromatin and discontinuous nuclear envelope (arrow). The cytoplasm contains abnormally swollen mitochondria (m), few rough endoplasmic reticulum (RER), lipid droplets (L), many vacuoles (v) and glycogen granules (g). Bile canaliculus (bc) is dilated. Kupffer cell (k) contains numerous vesicles and lysosomes varying in size and shape in the cytoplasm indicating its activity. (X 10200); (b) D21ACR showing abnormal hepatocyte with shrunken $(\mathrm{N})$ and peripheral condensation of chromatin (arrow). The cytoplasm contains abnormal shaped elongated mitochondria(m), rough endoplasmic reticulum (RER), some lipid droplets $(\mathrm{L})$, rarefication of the cytoplasm $\left(^{*}\right)$ appeared as electron lucent areas. Bilecanaliculus (bc) contains few microvilli. Notice Kupffer cell (K). (X 8500).

\section{3-Acrylamide Plus Vitamin E-Treated Group}

Hematoxylin and eosin staining: LM examination of sections from the liver of D1ACRVE albino rat showed normal central vein, blood sinusoids, portal vein and bile duct. Most hepatocytes were normal with polygonal large vesicular nuclei and acidophilic cytoplasm. Few hepatocytes had dark stained nuclei and others had pale nuclei. Few vacuoles were present in the cytoplasm of some hepatocytes. Multiple erythroid islands with intense hyperchromatic nuclei were present. Liver section of D21ACRVE subgroup showed apparently protection when compared to D21ACR. Hepatic architecture appeared similar to normal. Most of hepatocytes were normal with polygonal large vesicular nuclei and acidophilic cytoplasm however some hepatocytes had dark stained nuclei and others had pale nuclei. Few vacuoles were present in the cytoplasm of some hepatocytes. Normal central vein, blood sinusoids, portal vein and bile duct were observed (Figure 9).

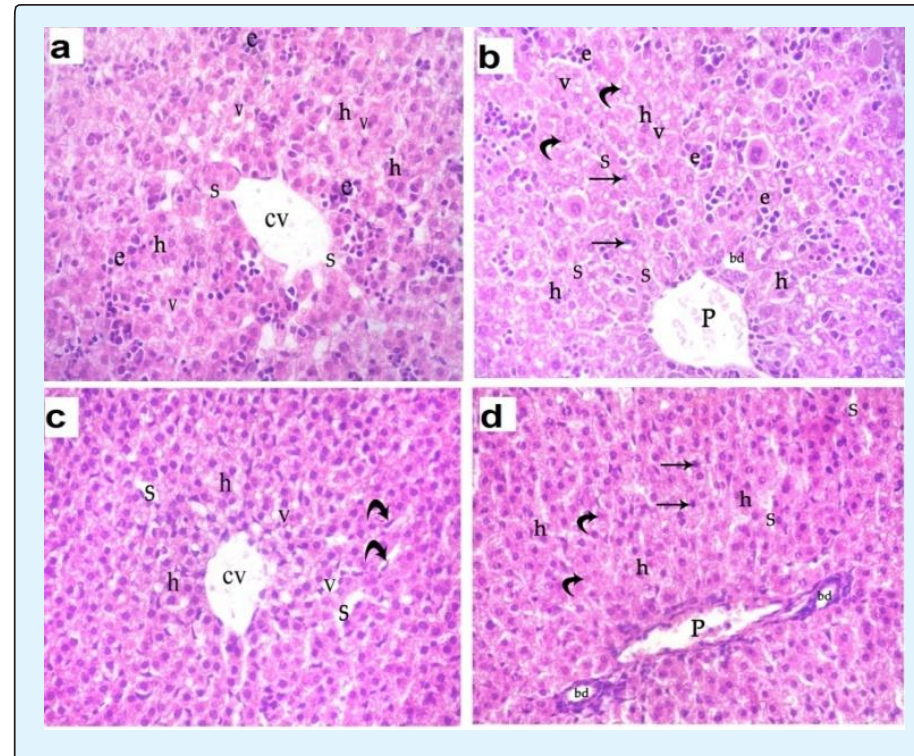

Figure 9: A photomicrograph of a liver section of ACR plus VE treated groups: (a) D1ACRVE showing normal or 


\section{Journal of Embryology \& Stem Cell Research}

slightly dilated central vein (cv), multiple small sized blood sinusoids (s), normal hepatocytes (h) with polygonal large vesicular nuclei and acidophilic cytoplasm. Few vacuoles (v) are present in the cytoplasm of some hepatocytes. Multiple erythroid islands (e) with intense hyperchromatic nuclei are noticed. (b) D1ACRVE showing normal or slightly dilated portal vein(p), normal bile duct (bd), multiple small sized blood sinusoids (s), normal hepatocytes (h). Some hepatocytes had dark stained nuclei (arrow) and others had pale nuclei (curved arrow). Few vacuoles (v) in the cytoplasm can be seen. Multiple erythroid islands (e). (c) D21ACRVE showing normal or slightly dilated central vein (cv), multiple small sized blood sinusoids(s), normal hepatocytes (h). Few vacuoles (v) are present in the cytoplasm of some hepatocytes. Some hepatocytes had dark stained nuclei (arrow). Kupffer cells (curved arrow) are seen in the lining of blood sinusoids. (d) D21ACRVE showing normal portal vein (p) and bile duct, multiple small sized blood sinusoids (s), normal hepatocytes (h). Some hepatocytes had dark stained nuclei (arrow) and others had pale nuclei (curved arrow).Few vacuoles (v) in the cytoplasm can be seen. (H\&E X400).

Mallory staining: Mallory stained sections of D1ACRVE subgroup showed few amount of collagen fibers around the central veins, blood sinusoids and portal area. Liver sections of D21ACRVEsubgroup showed also few amount of collagen fibers around the central veins, blood sinusoids and portal area (Figure 10).
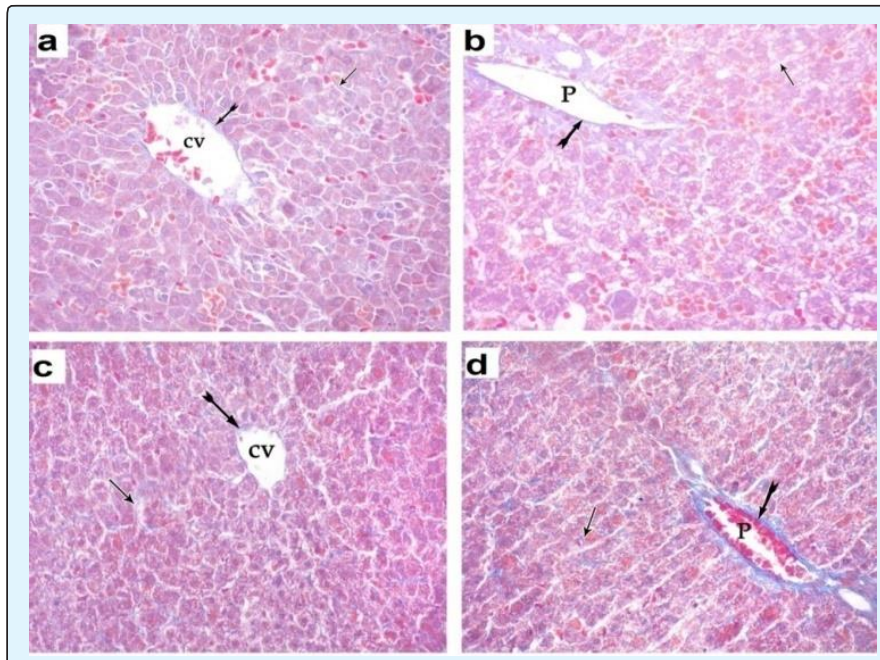

Figure 10: A photomicrograph of a liver section of ACR plus VE treated groups: (a,b) D1ACRVE showing few amount of blue colored collagen fibers (bifid tailed arrow) around the central vein (CV), in the lining of blood sinusoid (arrow) and in the portal area (P) (bifid tailed arrow). (c) D21ACRVE showing few amount of blue colored collagen fibers (bifid tailed arrow) around the central vein (CV) and in the lining of blood sinusoid (arrow). (d) D21ACRVE showing moderate amount of blue colored collagen fibers (bifid tailed arrow) in the portal area (P) and in the lining of blood sinusoid (arrow). (Mallory stain X400).

Immunohistochemical Staining: Liver section of D1ACRVE subgroup revealed mild positive cytoplasmic immune reaction to caspase 3.InD21ACRVE subgroup showed moderate positive cytoplasmic immune reaction to caspase 3 (Figure 11).

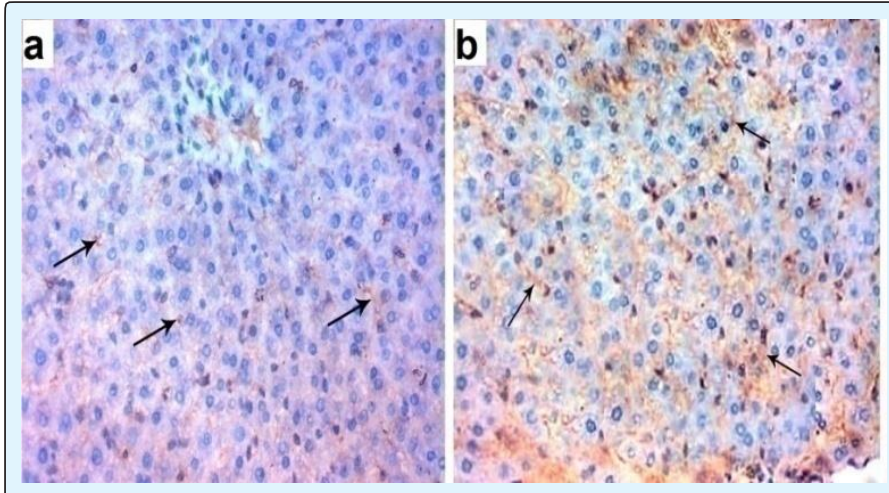

Figure 11: A photomicrograph of a liver section of ACR plus VE treated groups: (a) D1ACRVE and (b) D21ACRVE showing mild expression of cytoplasmic immune reaction for caspase 3 (arrow). (Immunoperoxidase technique for caspase 3 X 400).

EM Examination: EM study of liver sections of D1ACRVE showed nearly typical ultra structure of the liver. The hepatocytes appeared with euchromatic nuclei and intact nuclear envelope. The cytoplasm contained numerous mitochondria, rough endoplasmic reticulum, some lipid droplets and glycogen granules. Liver sections of D21ACRVE showed improvement of hepatic ultra structure when compared to D21ACR to subgroup.

Some hepatocytes appeared with euchromatic nuclei and intact nuclear envelope while some others had abnormally indented nucleus with in continuous nuclear envelope and peripherally condensed chromatin. The cytoplasm contained numerous mitochondria, rough endoplasmic reticulum, some lipid droplets, glycogen granules and some vacuoles. The hepatocytes were adjacent to each other with bile canaliculi containing (Figure 12). 


\section{Journal of Embryology \& Stem Cell Research}

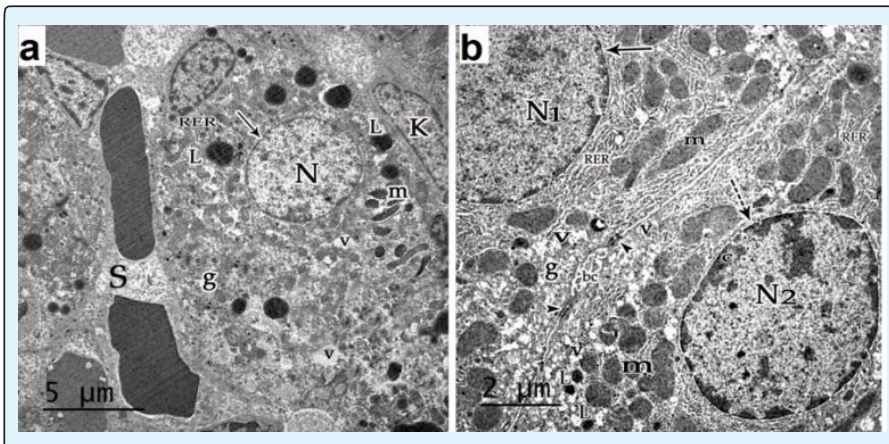

Figure 12: An EM micrograph of a section in the liver of ACR plus VE treated groups: (a) D1ACRVE showing normal hepatocyte with euchromatic nucleus (n) and intact nuclear envelope (arrow). The cytoplasm contains some abnormal mitochondria (m), few rough endoplasmic reticulum (RER), many lipid droplets (L), some vacuoles $(\mathrm{v})$ and glycogen granules $(\mathrm{g})$. Notice the nearby blood sinusoid (S) and Kupffer cell (k). (X 6800); (b) D21ACRVE showing upper normal hepatocyte with euchromatic nucleus (N1) and intact nuclear envelope (arrow). The lower hepatocyte had slightly indented nucleus (N2) with in continuous nuclear envelope (cut arrow) and peripherally condensed chromatin (c). The cytoplasm contained some abnormal mitochondria (m), rough endoplasmic reticulum (RER), many lipid droplets $(\mathrm{L})$, some vacuoles (v) and glycogen granules (g). Notice normal bile canaliculus (bc) containing microvilli and occluding junction around it (head arrow). (X 13600).

\section{Morphometry and Statistical Results}

Area Percentage of Collagen Fibers by One-Way Anova Test: The mean percentage area of collagen fibers in the negative control group was $0.65 \pm 0.21 \%$ while in the positive control group was $0.64 \pm 0.17 \%$. The mean percentage area of collagen fibers inD1ACR groups, D21ACR were $5 \pm 1.3 \%$ and $15 \pm 3.9 \%$, respectively.

The mean percentage area of collagen fibers inD1ACRVE and D21ACRVEsubgroups were $1.3 \pm 0.4 \%$ and $11.2 \pm 2.4 \%$, respectively. There was a highly significant difference between groups $(\mathrm{P}<0.05)$ (Table 1; Figure 13).

\begin{tabular}{|c|c|c|c|c|c|c|c|c|}
\hline \multirow{3}{*}{ cParameter } & Negative control & Positive control & D1 ACR & D21ACR & D1ACRVE & D21ACRVE & \multirow{2}{*}{ P } \\
\cline { 2 - 6 } & $\begin{array}{c}\text { mean } \\
\mathbf{\pm S D}\end{array}$ & $\begin{array}{c}\text { mean } \\
\mathbf{\pm S D}\end{array}$ & $\begin{array}{c}\text { mean } \\
\mathbf{\pm S D}\end{array}$ & $\begin{array}{c}\text { mean } \\
\mathbf{\pm S D}\end{array}$ & $\begin{array}{c}\text { mean } \\
\mathbf{\pm S D}\end{array}$ & $\begin{array}{c}\text { mean } \\
\mathbf{\pm S D}\end{array}$ & $\mathbf{F}$ & PD \\
\hline $\begin{array}{c}\text { Area } \\
\text { percentage }\end{array}$ & 1.04 & 1.028 & 18.2 & 20.2 & 4.8 & 6.2 & & \\
$\begin{array}{c}\text { Of immune reaction to } \\
\text { caspase 3 }\end{array}$ & $\mathbf{\pm}$ & $\mathbf{\pm}$ & $\mathbf{\pm}$ & $\mathbf{\pm}$ & $\mathbf{\pm}$ & $\mathbf{\pm}$ & 113.4 & $<0.001$ \\
\hline
\end{tabular}

Table 1: Statistical analysis of area percentage of collagen fibers by one-way ANOVA test.

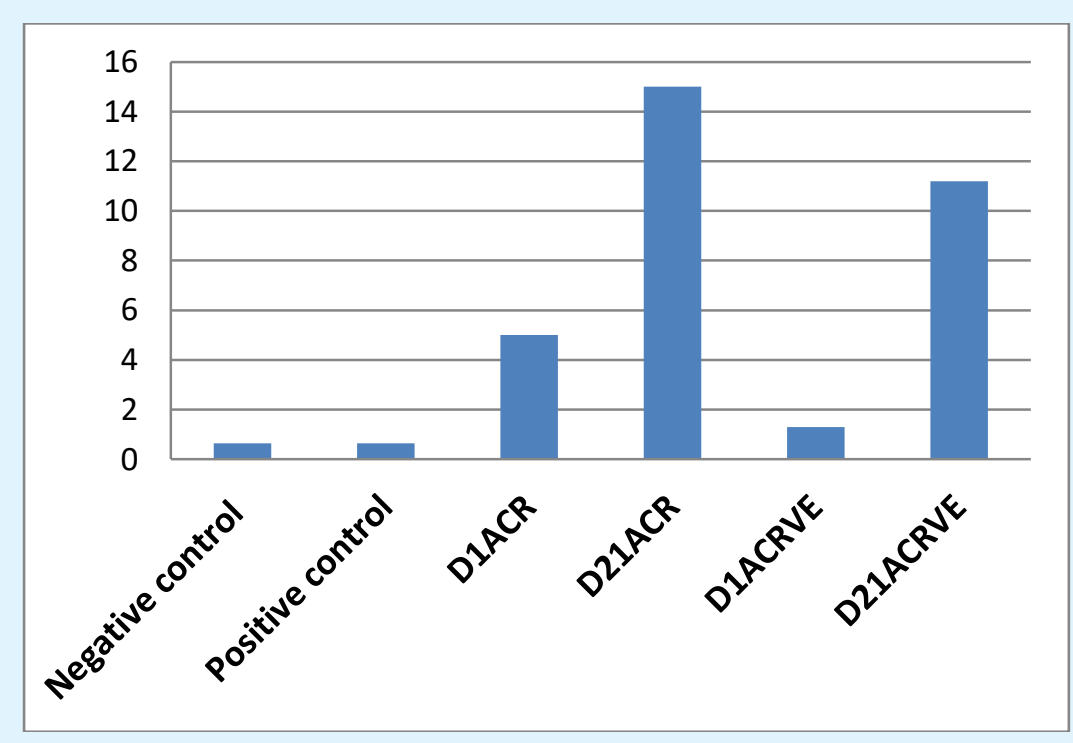

Figure 13: Comparison between mean values of area percentage of collagen fibers among the studied groups. 


\section{Journal of Embryology \& Stem Cell Research}

By least significant test, there was no significant difference between the negative and positive control subgroups $(P>0$. 05). Highly significant difference was found between control and D1ACR, D21ACR
andD21ACRVE $(\mathrm{P}<0.001)$. There was no significant difference between control and D1ACRVE subgroups $(\mathrm{P}>0.05)$ (Table 2).

\begin{tabular}{|c|c|c|c|c|c|c|}
\hline LSD & Negative control & Positive control & D1ACR & D21ACR & D1 ACRVE & $\begin{array}{c}\text { D21 } \\
\text { ACRVE }\end{array}$ \\
\hline Negative control & & $0.990 \mathrm{NS}$ & $<0.001$ & $<0.001$ & $0.301 \mathrm{NS}$ & $<0.001$ \\
\hline Positive control & $0.990 \mathrm{NS}$ & & $<0.001$ & $<0.001$ & $0.354 \mathrm{NS}$ & $<0.001$ \\
\hline D1ACR & $<0.001$ & $<0.001$ & & $<0.001$ & $<0.001$ & $<0.001$ \\
\hline D21ACR & $<0.001$ & $<0.001$ & $<0.001$ & & $<0.001$ & $<0.001$ \\
\hline D1ACRVE & $0.301 \mathrm{NS}$ & $0.354 \mathrm{NS}$ & $<0.001$ & $<0.001$ & & $<0.001$ \\
\hline D21ACRVE & $<0.001$ & $<0.001$ & $<0.001$ & $<0.001$ & $<0.001$ & \\
\hline
\end{tabular}

Table 2: The least significant difference of area percentage of collagen fibers between groups.

Area Percentage of Immune Reaction to Caspase 3 by One-Way ANOVA Test: The mean percentage area of immune reaction to caspase 3 was $1.04 \pm 0.49 \%$ and $1.028 \pm 0.38 \%$ in the negative and positive control subgroups respectively. The mean percentage areas of immune reaction to caspase 3 in ACR-treated groups D1 and D21 were $18.2 \pm 4.1 \%$ and $20.2 \pm 3.6 \%$, respectively. The mean percentage areas of immune reaction to caspase 3 in ACR plus VE-treated groups D1 and D21were $4.8 \pm 1.4 \%$ and $6.2 \pm 1.9 \%$, respectively. There wasa highly significant difference between groups $(\mathrm{P}<0.001)$ (Table3; Figure 14).

\begin{tabular}{|c|c|c|c|c|c|c|c|c|}
\hline & Negative control & Positive control & D1 ACR & D21ACR & D1ACRVE & D21ACRVE & \multirow{3}{*}{$\mathbf{F}$} & \multirow{3}{*}{$\mathbf{P}$} \\
\hline Parameter & mean & mean & mean & mean & mean & mean & & \\
\hline & \pm SD & $\pm \mathrm{SD}$ & \pm SD & \pm SD & \pm SD & \pm SD & & \\
\hline $\begin{array}{c}\text { Area } \\
\text { percentage } \\
\text { of } \\
\text { immune } \\
\text { reaction to caspase 3 }\end{array}$ & $\begin{array}{c}1.04 \\
\pm \\
0.49\end{array}$ & $\begin{array}{c}1.028 \\
\pm \\
0.38\end{array}$ & $\begin{array}{c}18.2 \\
\pm \\
4.1\end{array}$ & $\begin{array}{c}20.2 \\
\pm \\
3.6\end{array}$ & $\begin{array}{c}4.8 \\
\pm \\
1.4\end{array}$ & $\begin{array}{c}6.2 \\
\pm \\
1.9\end{array}$ & 113.4 & $<0.001$ \\
\hline
\end{tabular}

Table 3: Statistical analysis of area percentage of immune reaction to caspase 3 byone-way ANOVA test.

By least significant test, there was a highly significant difference between groups. There was no significant difference between the negative and positive control subgroups $(\mathrm{P}>0$. 05). There was a highly significant difference between control groups, D1ACR, D21ACR, D1ACRVE and D21ACRVE ( $P<0.001)$. There was a significant difference between D1ACR and D21ACR (P $<0.05$ ) (Table 4).

\begin{tabular}{|c|c|c|c|c|c|c|}
\hline LSD & Negative control & Positive control & D1ACR & D21ACR & D1ACRVE & D21ACRVE \\
\hline Negative control & & $0.990 \mathrm{NS}$ & $<0.001$ & $<0.001$ & $<0.001$ & $<0.001$ \\
\hline Positive control & $0.990 \mathrm{NS}$ & & $<0.001$ & $<0.001$ & $<0.001$ & $<0.001$ \\
\hline D1ACR & $<0.001$ & $<0.001$ & & 0.046 & $<0.001$ & $<0.001$ \\
\hline D21ACR & $<0.001$ & $<0.001$ & 0.046 & & $<0.001$ & $<0.001$ \\
\hline D1ACRVE & $<0.001$ & $<0.001$ & $<0.001$ & $<0.001$ & & $0.175 \mathrm{NS}$ \\
\hline D21ACRVE & $<0.001$ & $<0.001$ & $<0.001$ & $<0.001$ & $0.175 \mathrm{NS}$ & \\
\hline
\end{tabular}

Table 4: The least significant difference test of area percentage of caspase 3 between different groups. 


\title{
Journal of Embryology \& Stem Cell Research
}

There was some improvement but not full protection in the VE-added cases in comparison with the control and

\author{
ACR-treated cases (Figures $13 \& 14$ ).
}

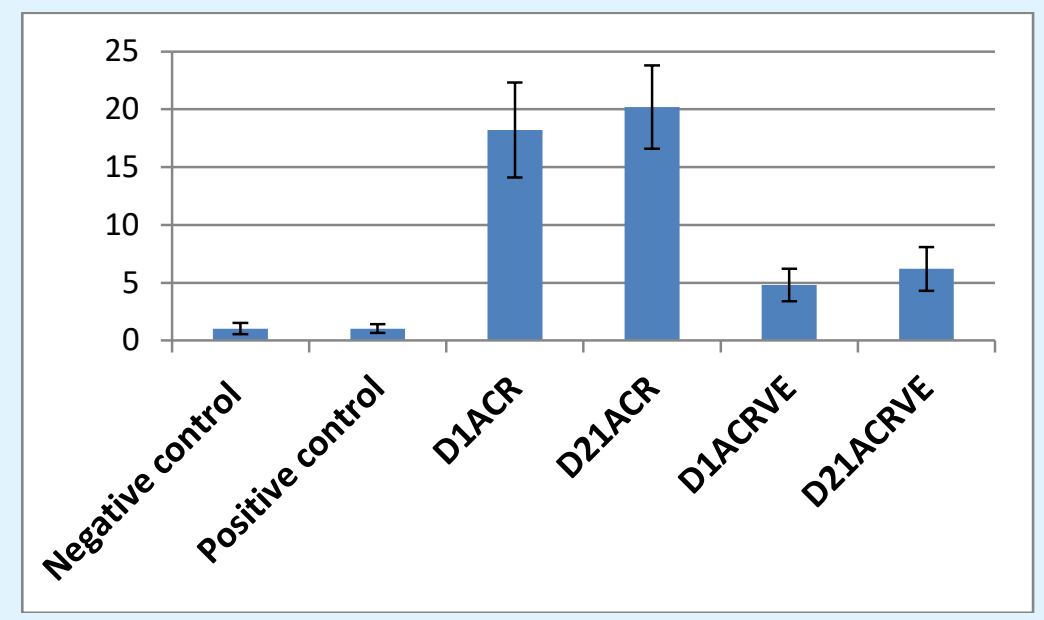

Figure 14: Comparison between mean values of area percentage of cytoplasmic reaction to caspase 3 among the studied groups.

\section{Discussion}

The ACR is transferred via breast milk from lactating mother to the baby. Mothers consuming high ACR diet can transfer up to $10 \mu \mathrm{g}$ of acrylamide to her baby when the baby daily consumption is about $500 \mathrm{ml}$ of breast milk. Mothers who eat little to moderate amounts of ACR containing foods, likes chips or potato may still transfer 2 $\mu \mathrm{g}$ of acrylamide to her baby [18]. In the current study, ACR was administered orally during gestation and weaning.

In the present work, light microscopic examination of sections from the liver of control group D1 showed that the hepatocytes were normal with characteristic polygonal large vesicular nuclei and acidophilic cytoplasm, scattered erythroid islands (small islands of hematopoietic cells). The erythroid cells were characterized by intense hyperchromasia in their nuclei in contrast to pale nuclei of hepatocytes. The central vein and blood sinusoids were normal in size. These results are in accordance with $\mathrm{Li}$, et al [19] who mentioned that during of active liver hematopoiesis stage at prenatal life, macrophages migrate from sinusoids to the parenchyma to form erythroid islands.

The liver of the control albino rat's groupsD21 revealed that each hepatic lobule was formed of radiating tightly packed cords of hepatocytes from the central vein. These cords were composed of polygonal hepatocytes with rounded vesicular nuclei and acidophilic cytoplasm. Some cells were binucleated. Blood sinusoids were present in between hepatocyte cords. The portal areas containing bile ducts and blood vessels were seen. These results are in agreement with those described by Baratta, et al. [20] who declared that the characteristic histological architecture of liver is present by the end of third week.

In the present work, few collagen fibers around central vein and in portal area were detected by Mallory's trichrome stained liver sections of D1andD21 control subgroups.These findings are in agreement with that of Ali, et al. [21].

In the present work, liver sections of all control subgroups showed negative immune reaction for caspase 3 in the cytoplasm of hepatocytes due to decreased apoptotic cells in normal hepatic tissue. These findings were in accordance with those described by El Kalawy, et al. [22].

In the present work, EM study of the control subgroups showed that the hepatocytes appeared with euchromatic nuclei with intact nuclear envelope. The cytoplasm contained numerous rough endoplasmic reticula, mitochondria, glycogen granules and some lipid droplets. The hepatocytes were adjacent to each other with bile canaliculi containing microvilli and occluding junctions were observed. These results were in agreement with Mohamed and Selim [23] who mentioned that normal 


\section{Journal of Embryology \& Stem Cell Research}

ultra structure of the liver was characterized by abundant rough endoplasmic reticulum and mitochondria in the cytoplasm of hepatocytes.

In the present work, light microscopic examination of the liver of group II (ACR-treated) D1ACR showed a change of normal hepatic structure. Hepatocytes had pale vacuolated cytoplasm with deeply stained shrunken nuclei. There were dilated congested central vein and blood sinusoids and dilated congested Portal vein. On the other hand, D21ACR showed the previous changes but more extensive. periportal inflammatory cells infiltration, Congestion and detachment of endothelial lining of portal veins were also demonstrated. Bile canaliculi Proliferation were also noticed. So, the most damaging effect of acrylamide was observed in the ACR-treated subgroup (D21) due to prolonged exposure to ACR. These ACR-induced changes are in agreement with the reports of Rady and Osman [24] who observed hepatoxicity following the ingestion of excess ACR containing diet.

Also, the present work showed congested portal and central veins. Distinct blood vessel dilation could suggest metabolic disturbances in the liver, thus indicating a potential toxic effect of ACR on the liver during the fetal period. These toxic results of ACR on the liver are in accordance with that of Kurebayashi and Ohno [25] who reported that once ACR was absorbed, it was conjugated by glutathione -s-transferase to $\mathrm{N}$-acetyl-s-(3-amino-3 oxopropyl) cysteine or reacted with cytochrome P450 to produce glycidamide. This led to the depletion of glutathione, which is considered an important antioxidant enzyme. Therefore, oxidative damage might occur and interfere with cell function and promote cell death.

The above mentioned results were explained by Mohamed and Selim [23] who said that the hepatotoxic action of ACR is due to cell respiration disorders and disturbances in intracellular and extracellular transport. As a consequence, the hepatocytes or their cytoplasmic organelles can be damaged. This damage is manifested as parenchymal degeneration, necrosis of hepatocytes, or disorders in the activity of metabolic enzymes.

Gutowsk, et al. [26] suggested that vacuolated cells may be macrophages containing many lipid droplets (foam cells). They added that the increased levels of ROS lead to modification of low density lipoproteins (LDLs) with formation of oxidized form (OX-LDLs) which are internalized by the macrophages and is implicated in the formation of foam cells.
In the current study, vascular congestion and dilatation revealed in treated rats could be explained by activation of Kupffer cell with subsequent elevation of production of nitric oxide considered contributing factor for vascular dilatation [27]. $\mathrm{Hu}$, et al. [28] confined the dilated and congested central vein and blood sinusoids with separation of the endothelial lining of the central vein finding to portal hypertension. On the other hand, Puche, et al. [29] attributed sinusoidal dilatation to activation of perisinusoidal cells that had contractile properties.

In the present work, some hepatocytes in ACR-treated group have darkly stained nuclei. This finding is in accordance with Taylor et al. [30] who stated that hyperchromatic nuclei were a degenerative change denoting apoptosis. In the liver, both Kupffer cells and hepatic stellate cells can phagocytose apoptotic bodies [31].

In the current work, Mallory-stained sections of ACRtreated group revealed marked increase in collagen fibers around the blood vessels. The excess fibrosis was prominent in D21 than D1 due to chronic exposure to toxic effect of ACR. This finding is in accordance with Treesh et al. [32] who stated that collagen synthesis is enhanced by ROS which promote hepatic fibrosis as they induce proliferation of hepatic stellate cells. Activated stellate cells leaded to the deposition of collagen type I.

The marked periportal cellular infiltration noticed in this study was explained by Fahmi, et al. [33] who stated that cytokines produced by neutrophils and activated monocytes are the main modulators of inflammatory response. These cytokines can stimulate the production of prostaglandins E2 and collagenase and thus are believed to be involved in tissue damage with subsequent fibrosis.

Detachment of endothelial lining of portal veins was noticed in the current work. This finding is in accordance with Martin [34] who mentioned that the high level of neutrophils number in the blood capillaries could damage their epithelium lining by secreting several injurious substances such as lysosomal enzymes and oxygen derived free radicals.

In the present work, bile ductules proliferation was evident on LM examination. Suriawinata and Thung [35] stated that a ductular proliferation is a reactive response to liver injuries like drugs, toxins and viruses. Micalopoulos et al. [36] reported that of bile ducts proliferation could be produced by trans-differentiation of hepatocytes cells into biliary cells or due to bile salt 


\section{Journal of Embryology \& Stem Cell Research}

stasis which could be attributed to produce the mitogenic effect on biliary epithelial cells. They added that passage of low viscosity bile through the bile canaliculi is active process need energy, so it can be injured by several hepatotoxins especially in early stages before producing histological changes.

In the present work by EM examination of D1ACR and D21ACR sub groups showed electron-lucent areas of the cytoplasm of hepatocytes, with many vacuoles and lipid droplets. Some of them had shrunken electron-dense nuclei, whereas others had peripheral condensation of the nuclear chromatin. More destruction was in D21 than D1. These results match that of Mohamed and Selim [23] who mentioned that electron microscope examination of acrylamide-treated groups showed degenerative changes in liver tissue. These changes appeared as shrunken nuclei, rarified cytoplasm and vacuolization. Suriawinata and Thung [35] mentioned that Cytoplasmic vacuolation could be attributed to mitochondrial dysfunction with ATP depletion. The mitochondrial degeneration probably occurs because of loss of integrity of plasma membrane followed by electrolyte imbalance with subsequent massive influx of water and sodium.

In the current study, LM examination of the liver sections of ACR plus VE-treated subgroups showed improvement in hepatic architecture. Central veins and blood sinusoids are normal or showed mild dilation. Most of hepatocytes are normal with polygonal large vesicular nuclei and acidophilic cytoplasm. While some of them had deeply stained nuclei and vacuolated cytoplasm. The portal area was normal with slight congestion of portal vein. Mallory-stained sections showed mild increased deposition of collagen fibers around the central veins and blood sinusoids and mild increased deposition of collagen fibers in portal area. In addition, weak positive cytoplasmic immune reaction to caspase 3(mild expression) was noticed. Moreover, EM study of the ACR plus VE-treated sub groups showed nearly typical normal ultra structure of the liver.

These results are in agreement with AL-Rasheed et al. [37] who mentioned that supplementation with VE produced hepato-protection in the form of preserved hepatic architecture also they stated that the level of apoptosis was markedly decreased and there was minimum collagen fibers in portal area and around blood vessels. Preserved normal histological architecture structure in ACR and VE-treated subgroups is attributed to VE natural anti-inflammatory and antioxidant properties which ameliorate the oxidative damage by trapping of reactive oxygen and nitrogen species, and also by down-regulating of inflammatory markers as CRP and IL-6 [10]. In contrast with these results, Rahangadale, et al. [38] concluded that VE doesn't alleviate the ACRinduced toxicity during active exposure. Moreover, Erdemli, et al. [39] have performed biomedical analysis and concluded that the liver affected by the ACR is that of the pregnant mothers not of their fetuses. They added that the mother liver acts as a filter preventing the ACR from reaching their fetuses. On contrary with these results, the current study showed hazard affection of liver of the pubs by oral administration of ACR. This discrepancy might be attributed to its administration to the pregnant mothers as well as to their pubs during weaning in the current investigation.

\section{Conclusion}

The current study suggested that ACR oral administration could cause hazardous impact in the liver of albino rats. Fortunately, concomitant receiving of VE might provide some protection to the liver and attenuate the ACR-induced hepatotoxicity. Further studies are recommended to test the long-run protective role of $\mathrm{VE}$ after cessation of ACR exposure.

\section{Conflict of Interest}

The authors declare that they have no conflicting financial interests.

\section{References}

1. Akharaiyi F, Boboye B, Adetuyi F (2012) Hepatoprotective effect of ethanol leaf extract of cnestis ferruginea on Swiss Albino Mice Induced with Paracetamol. Int Res J Pharmaceuticals 2(4): 120-126.

2. Isenberg JS, Kamendulis LM, Smith JH, Ackley DC, Pugh GJ, et al. (2000) Effects of Di-2-ethylhexyl phthalate (DEHP) on gap-junctional intercellular communication (GJIC), DNA synthesis, and peroxisomal beta oxidation (PBOX) in rat, mouse, and hamster liver. Toxicol Sci 56(1): 73-85.

3. Lo Pachin RM (2004) the changing view of acrylamide neurotoxicity, Neurotoxicology 25(4): 617-630.

4. Rommens CM, Yan H, Swords K, Richael C, Ye J (2008) Low acrylamide French fries and potato chips. Plant Biotechnol J 6(8): 843-853. 


\section{Journal of Embryology \& Stem Cell Research}

5. Hagmar L, Törnqvist M, Nordander C, Rosén I, Bruze $M$, et al. (2001) Health effects of occupational exposure to acrylamide using hemoglobin adducts as biomarkers of internal dose. Scand J Work Environ Health 27(4): 219-226.

6. Tareke E, Rydberg P, Karlsson P, Eriksson S, Törnqvist M (2002) Analysis of acrylamide, a carcinogen formed in heated foodstuffs. J Agric Food Chem 50(17): 4998-5006.

7. Larion S, Khurana S (2018) Clinical studies investigating the effect of vitamin $\mathrm{E}$ therapy in patients with NASH. Clinical liver disease 11(1): 1621.

8. Mansour MK, Ibrahim EM, Maha M, El-Kholy, Sahar A (2008) Antioxidant and histopathological effect of catechin and neem leaves extract in acrylamide toxicity of rats. Egypt J Comp and Clinic Pathol 21(1): 290-313.

9. El-Bohi KM, Moustafa GG, El sharkawi NI, Sabik LM (2011) Genotoxic effects of Acrylamide in Adult Male Albino Rats Liver. Journal of American Science 7(1): 1097-1108.

10. El Hadi H, Vettor R, Rossato M (2018) Congenital vitamin E defiency. In: Preedy VR, Patel VB (Eds.), handbook of famine, starvation and nutrient deprivation. Springer International Publishing AG, Basel, Switzerland; pp: 1-18.

11. Tyla RW, Friedman MA, Losco PE, Fisher LC, Johnson $\mathrm{KA}$, et al. (2000) Rat two-generation reproduction and dominant lethal study of acrylamide in drinking water. Reproduct Toxicol (Elmsford NY) 14: 385-401.

12. Shati AA (2014) Ameliorative effect of vitamin E on potassium dichromate-induced hepatotoxicity in rats. Journal of King Saud University-Science 26(3): 181189.

13. Sengupta P (2013) The Laboratory Rat: Relating Its Age with Human's. Int J Prev Med 4(6): 624-630.

14. Hegazy R, Hegazy A (2015) Hegazy' Simplified Method of Tissue Processing (Consuming Less Time and Chemicals). Ann of Int Med \& Den Res 1(2): 5761.

15. Bancroft J, Gamble M (2008) Theory and Practice of Histological Techniques. 6th (Edn), Churchill Livingston, pp: 165-175.

Hegazy AA, et al. Effect of Administration of Acrylamide and Possible Protective Role of Vitamin E on Postnatal Rat Liver Structure. J Embryol Stem Cell Res 2018, 2(2): 000114.
16. Woods A, Stirling J (2002) Electron Microscopy: The preparative techniques. In: Bancroft J, Gamble M (Eds.), Theory and Practice of Histological Techniques. $5^{\text {th }}$ (Edn.), Churchill Livingston, New York, Edinburgh, London, pp: 682-700.

17. Field A (2013) Discovering statistics using IBM SPSS. Sage, New Delhi.

18. Sörgel F, Weissenbacher R, Kinzig-Schippers $M$, Hofmann A, Illauer M, et al. (2002) Acrylamide: increased concentrations in homemade food and first evidence of its variable absorption from food, variable metabolism and placental and breast milk transfer in humans. Chemotherapy 48(6): 267-274.

19. Li D, Wang GY, Liu ZF, Shi YX, Zhang H, et al. (2004) Macrophage associated erythropoiesis and lymphocytopoiesis in mouse fetal liver: ultrastructural and ISH analysis. Cell Biology International 28(6): 457-461.

20. Baratta JL, NgoA, LopezB, KasabwallaN, Longmuir KJ, (2009) Cellular Organization of Normal Mouse Liver: A Histological, Quantitative Immunocytochemical, and Fine Structural Analysis. Histochem Cell Biol 131(6): 713-726.

21. Ali SA, Rizk MZ, Abdelazim SA, Darwish HA, Kadry MO (2015) Role of potent antioxidants in regulation of smad- 2 transcription and TGF- $\beta 1$ signaling in Nano sized titanium dioxide-induced oxidative injury in mice liver. International Journal of Pharmacotherapy 5: 17-26.

22. El Kalawy SA, Mostafa A, Abdelafattah LI, Kamar SS (2017) Histological and Immunohistochemical study of the effect of omega- 3 fatty acid on ifosfamideinduced liver injury adult male albino rat. JMH 1(2): 135-145.

23. Mohamed DA, Selim SA (2012) Effect of perinatal acrylamide exposure on the liver of albino rat offspring: light and electron microscopic study. Egyptian journal of Histology 35(3): 371-382.

24. Rady MI, Osman AM (2006) Histological alterations in the liver of mother rats and its weanlings fed on fried bread and the protective effect of curcumin. J Egypt Soc Toxicol 35: 19-35.

25. Kurebayashi H, Ohno Y (2006) 1-Metabolism of acrylamide to glycidamide and their cytotoxicity in 


\section{Journal of Embryology \& Stem Cell Research}

isolated rat hepatocytes: protective effects of GSH precursors. Arch Toxicol 80(12): 820-828.

26. Gutowsk I, Baranowska-Bosiack I, Siennick A, Rybicka M, Baśkiewicz M, et al. (2011) Fluoride effects on cholesterol influx and expression of the scavenger receptor cd36 in thp1 differentiated monocyte/macrophage cells. Research Report Fluoride 44(3): 135-142.

27. Roberts RA, Ganey PE, Ju C, Kamendulis LM, Rusyn I, et al. (2007) Role of the Kupffer Cell in Mediating Hepatic Toxicity and Carcinogenesis. Toxicological Sciences 96(1): 2-15.

28. Hu LS, George J, Wang JH (2013) Current concepts on the role of nitric oxide in portal hypertension. World J Gastroenterol 19(11): 1707-1717.

29. Puche J, Saiman Y, Friedman S (2013) Hepatic Stellate Cells and Liver Fibrosis. Compr Physiol 3(4): 14731492.

30. Taylor RC, Cullen SP, Martin SJ (2008) Apoptosis: controlled demolition at the cellular level. Nat Rev Mol Cell Biol 9(3): 231-241.

31. Wang S, Pacher $\mathrm{P}$, De Lisle RC, Huang H, Ding WX (2016) A Mechanistic Review of Cell Death in AlcoholInduced Liver Injury. Alcohol Clin Exp Res 40(6): 1215-1223.

32. Treesh S, El Jaafari H, Darmun E, Abu-Aisha A (2014) Histological study on the effect of formaldehyde on mice liver and kidney and possible protective role of selenium. Journal of Cell and Tissue Research 14(2): 4201-4209.
33. Fahmi AN, Shehatou GS, Shebl AM, Salem HA (2016) Febuxostat protects rats against lipopolysaccharideinduced lung inflammation in a dose-dependent manner. Naunyn Schmiedebergs Arch Pharmacol 389(3): 269-278.

34. Martin TR (2002) Neutrophils and lung injury: Getting it right. J Clin Invest 110(11): 1603-1605.

35. Suriawinata AA, Thung SN (2011) Hepatocyte degeneration, death and regeneration. In: Liver Pathology, an Atlas and Concise Guide. New York: Demos Medical Publishing, pp: 19-20.

36. Micalopoulos GK, Barua L, Bowen WC (2005) Transdifferentiation of rat hepatocytes into biliary cells after bile duct ligation and toxic biliary injury 41(3): 535-544.

37. Al-Rasheed NM, Al-Rasheed NM, Abdel Baky NA, Faddah LM, Fatani AJ, et al. (2014) Prophylactic role of $\alpha$-lipoic acid and vitamin E against zinc oxide nanoparticles induced metabolic and immune disorders in rat's liver. Eur Rev Med Pharmacol Sci 18(12): 1813-1828.

38. Rahangadale S, Kurkure N, Prajapati B, Hedaoo V, Bhandarkar AG (2012) Neuroprotective effect of vitamin e supplementation in wistar rat treated with acrylamide. Toxicol Int 19(1): 1-8.

39. Erdemli ME, Altinoz E, Aksungur Z, Turkoz Y, Dogan Z, et al. (2017) Biochemical investigation of the toxic effects of acrylamide administration during pregnancy on the liver of mother and fetus and the protective role of vitamin E. J Matern Fetal Neonatal Med 30(7): 844-848. 